ren/2014/fortschreibung-up-kritis.pdf?_blob=publicationFile) heruntergeladen werden. Unternehmen, die Kritische Infrastrukturen betreiben, sind herzlich zur Mitwirkung eingeladen. Die Zuordnung lässt sich anhand der Sektoreneinteilung für Kritische Infrastrukturen ableiten. Die Geschäftsstelle des UP KRITIS im Bundesamt für Sicherheit in der Informationstechnik steht für Aufnahmeanträge und Rückfragen jeglicher Art gern zur Verfügung.

\section{IT-Gipfel wird Dialogplattform für die Umsetzung der Digitalen Agenda}

Bundesminister Gabriel hat am 21.07.2014 mit führenden Wirtschaftsvertretern die Schwerpunktthemen des nächsten IT-Gipfels am 21. Oktober 2014 in Hamburg abgestimmt und die Neuausrichtung des Gipfel-Prozesses auf die Digitale Agenda der Bundesregierung in den nächsten Jahren beraten.

Bundesminister Gabriel: „Mir ist wichtig, dass wir bei den Fragen der Digitalisierung der Wirtschaft unsere traditionell starken Anwenderindustrien wie Maschinenbau, Elektrotechnik und Automobilbau stärker einbeziehen. Wir brauchen neben der Expertenebene eine zweite Ebene, auf der wir uns über die politischen Rahmenbedingungen der smarten Produktion, der Verlagerung von Daten und Anwendungen ins Internet oder der Bearbeitung und Analyse riesiger Datenmengen in Echtzeit zügig und effektiv verständigen können."

Gabriel kündigte dazu die Einrichtung der „Dialogplattform Industrie 4.0" an. Diese soll den Austausch der führenden Akteure aus Wirtschaft, Wissenschaft, Gesellschaft und Regierung über die notwendigen politischen und gesellschaftlichen Rahmenbedingungen für die großen Trends von Industrie 4.0 und die Digitalisierung der Wirtschaft fördern und beschleunigen.

In der strategischen Arbeitsgruppe 1 des IT-Gipfels, die von Bundeswirtschaftsminister Gabriel und BITKOM-Präsident Prof. Kempf geleitet wird, bestand Einigkeit, die Themen Industrie 4.0, Young IT, Arbeiten in der digitalen Welt und IT-Sicherheit sowie digitaler Content auf dem Hamburger Gipfel in den Vordergrund zu stellen. Dort sollen auch konkrete Umsetzungsprojekte zur Digitalen Agenda im Bereich Junge digitale Wirtschaft und Digitalisierung der Wirtschaft vorgestellt werden.

\section{Sicherheitsverantwortliche und Management: Kommunikation verbessern}

Websense und das Ponemon Institute haben am 21.07.2014 eine neue Sicherheitsstudie vorgestellt. Sie offenbart erhebliche Mängel in der Kommunikation zwischen Sicherheitsverantwortlichen und Management. Außerdem herrscht große Unzufriedenheit mit den vorhandenen Security-Systemen.

Für die aktuelle, von Websense in Auftrag gegebene Untersuchung „Roadblocks, Refresh, \& Raising the Human Security IQ" hat das Ponemon Institute weltweit rund 5.000 Verantwortliche für IT-Sicherheit in 15 Ländern befragt, darunter England, Deutschland, Frankreich, Italien, die Niederlande, Schweden und die USA.

Der jetzt vorgestellte Bericht offenbart große Mängel in der Kommunikation zwischen Sicherheitsverantwortlichen und Führungskräften sowie einen starken Wunsch nach der Überarbeitung vorhandener Security-Systeme. Außerdem, so die Studie, verfügten die Mitarbeiter der Unternehmen nur über begrenztes Sicherheitswissen. Nachfolgend sind die wichtigsten Ergebnisse der Untersuchung zusammengefasst.

Kommunikationsmängel zwischen Sicherheitsverantwortlichen und Führungskräften:

- 31\% der für Security zuständigen Teams sprechen nie mit dem Management über Cyber-Sicherheit;

- Von denjenigen, die es doch tun, führen fast ein Viertel (23\%) solche Gespräche nur einmal im Jahr und weitere 19\% nur einmal pro Halbjahr. Lediglich $11 \%$ sprechen vierteljährlich mit ihrem Management und gerade einmal 1\% tut dies wöchentlich.

Security-Teams wünschen sich komplette Aktualisierung der Sicherheits-Systeme:

- 29\% der Studienteilnehmer würden die vorhandenen Security-Systeme ihrer Unternehmen komplett überarbeiten - wenn sie die nötigen Ressourcen und Möglichkeiten dazu hätten;

- Mit $47 \%$ ist fast die Hälfte der Befragten regelmäßig über das Schutz-Level enttäuscht, das erworbene Sicherheits-Lösungen am Ende tatsächlich bieten. Lediglich 12\% sind mit ihren Security-Systemen zufrieden;

- 56\% der Studienteilnehmer glauben, dass ein Fall von Datendiebstahl im Unternehmen zu einem Wechsel des Sicherheitsanbieters führen würde;

- Advanced Persistent Threats und Daten-Exfiltration werden von den Sicherheitsverantwortlichen als größte Bedrohungen gesehen;

- 49\% der Befragten gaben an, in den nächsten zwölf Monaten signifikante Investitionen und Anpassungen für ihre Verteidigungsmaßnahmen zu planen.

Sicherheits-Know-how der Mitarbeiter wird zu wenig gefördert:

- Nur 38\% der Befragten glauben, dass ihre Unternehmen ausreichend in Personal und Technologien investieren, um die avisierten Cyber-Security-Ziele zu erreichen;

- 52\% der Unternehmen bieten keine entsprechenden Schulungen für ihre Mitarbeiter an;

- Weniger als die Hälfte der befragten Sicherheitsverantwortlichen (42\%) haben im Rahmen ihrer derzeitigen Position einen Threat-Modelling-Prozess durchgeführt. Die große Mehrheit dieser Gruppe (94\%) fand dies für den Umgang mit Cyber-Risiken wichtig;

- Unter den Ereignissen, die nach Ansicht der Studienteilnehmer das Management ihres Unternehmens dazu bringen würden, mehr Mittel für Security-Initiativen bereitzustellen, nehmen folgende Fälle die Spitzenplätze ein: Verlust von geistigem Eigentum (67\%), Diebstahl von Kundendaten (53\%) und Umsatzeinbußen durch Systemausfälle (49\%).

„Die globale Untersuchung des Ponemon Institute zeigt mangelnde Kommunikation, fehlendes Know-how und unzulängliche Systeme bei der Abwehr von Cyber-Bedrohungen. Das ermöglicht Kriminellen auf der ganzen Welt, Unternehmen erfolgreich zu attackieren“, sagt Michael Rudrich, Regional Director Central Europe \& Eastern Europe bei Websense in München. „Es ist nicht überraschend, dass viele Sicherheitsverantwortliche von ihren Security-Lösungen enttäuscht sind. Sie nutzen häufig noch Altsysteme, 